\title{
Contraceptive use in Adolescents with Perinatally and Behaviorally Acquired Human Immunodeficiency Virus Infection Seen in a Public Los Angeles County Clinic
}

\author{
Jessica Atrio ${ }^{1,2}$, Kyle Graham ${ }^{2}$, Hita Vora ${ }^{2,3}$, Eva Operskalski ${ }^{2,4}$ and Andrea Kovacs ${ }^{2,4}$ \\ ${ }^{1}$ Department of Obstetrics and Gynecology and Virology, University of Southern California Keck School of Medicine, Los Angeles, CA, USA \\ 2University of Southern California Keck School of Medicine, Los Angeles, CA, USA \\ ${ }^{3}$ Southern California Clinical and Translational Science Institute, USA \\ ${ }^{4}$ Maternal Child and Adolescent/Adult Center for Infectious Diseases, USA
}

\begin{abstract}
Objective: Primary aim is to compare contraception practices between adolescent females aged 15-25 years with perinatally vs. behaviorally acquired HIV infection

Design: A retrospective, pilot, chart review of 33 HIV positive adolescent females 15-25 years old, with one or more clinic visits at the Maternal Child and Adolescent Center for Infectious Diseases and Virology from January 1 , 2010 to December 31, 2012 was conducted. Secondary outcomes included number of pregnancies, changes in contraceptive method, and condom use. Adolescents with perinatally and behaviorally acquired HIV infection were compared; and both groups were compared to the United States Centers for Disease Control national statistics.

Results: Eighteen (55\%) perinatally and $15(45 \%)$ behaviorally HIV infected adolescents were studied. Depo medroxyporgesterone Acetate (DMPA) was the most commonly used contraceptive by the perinatal group $(n=7,39 \%)$ and the second most common method in the behaviorally infected group $(n=4,27 \%)$. Condoms were the second most commonly used method among perinatally infected adolescents $(n=4,22 \%)$. Condoms in addition to a hormonal contraceptive was used by $5(28 \%)$ perinatal and $5(33 \%)$ of the behavioral adolescents. None of the behavioral group used condoms as the exclusive method of contraception. Long Acting Reversible Contraception (LARC) was the most commonly used method by the behavioral group $(n=5,33 \%)$. The average number of pregnancies was 2.8 (range 1-5) among adolescents with behaviorally acquired HIV and. 0.4 (range 0-2) among adolescents with perinatally acquired infection $(P<0.001)$.

Conclusion: In this small pilot study, perinatally infected adolescents were more likely to use DMPA or condoms as compared to behaviorally infected adolescents, who were more likely to use LARC or DMPA though these differences were not statistically significant given the small sample size. Future larger studies should explore pregnancy and contraception in these distinct populations
\end{abstract}

Keywords: HIV; AIDS; Contraception; Family planning; Parity; Gravity; Teen pregnancy; Heterosexual infection; Perinatal infection; Adolescents; Girls; Women

\section{Introduction}

In the United States there were approximately 76,400 HIV positive adolescent age 13 to 24 years of age in 2009 , and approximately 77 infants acquired HIV through perinatal transmission [1]. In 2006 approximately $8,700 \mathrm{HIV}$ positive women gave birth, a $30 \%$ increase from the number of women in 2000 [2]. HIV positive adolescent females are a unique population with compelling sexual and reproductive health needs. Their contraception practices have significant social ramifications in terms of risk of heterosexual transmission, re-infection and vertical transmission [3]. There are limited data that explore contraceptive choice and decision-making among these young women [4,5]. Some research has demonstrated that HIV positive young women engage in reproductive decision-making that is similar to HIV negative teens [5]. Observational studies have demonstrated a similar desire among HIV positive and negative adolescents to prevent or delay pregnancy, and similar rates of sexual début by age 17 [6].

HIV positive adolescents may not represent a homogeneous population with regard to contraceptive practices and risk for unintended pregnancy. The behavior patterns of perinatally vs. behaviorally infected HIV positive adolescents may reflect differences in their contraceptive needs; some may want to avoid pills, or they may have different desired life time pregnancies or have different concerns regarding disclosure and transmission. Additionally, these two groups may not exhibit similar sexual behaviors or risk profiles [7]. The majority of adolescents in the United States are sexually active by 18 years of age [8]. In the transition from puberty to adulthood, young women are navigating personal independence, self-identity, values, new legal status, and responsibilities. Sexual health and healthrisk behaviors that are established in adolescents often extend into adulthood and are interrelated [7].

Understanding the contraceptive practices of perinatally and behaviorally HIV-infected adolescents would help providers to identify potential interventions for unmet needs. Dual prevention with condoms

*Corresponding author: Jessica Atrio, Department of Obstetrics and Gynecology and Virology, University of Southern California Keck School of Medicine, Los Angeles, CA, USA, Tel: (917) 721-6155; E-mail address: jessicaatrio@gmail.com

Received August 02, 2013; Accepted September 13, 2013; Published September 18, 2013

Citation: Atrio J, Graham K, Vora H, Operskalski E, Kovacs A (2013) Contraceptive use in Adolescents with Perinatally and Behaviorally Acquired Human Immunodeficiency Virus Infection Seen in a Public Los Angeles County Clinic. J AIDS Clin Res 4: 244 doi: 10.4172/2155-6113.1000244

Copyright: (c) 2013 Horner P, et al. This is an open-access article distributed unde the terms of the Creative Commons Attribution License, which permits unrestricted use, distribution, and reproduction in any medium, provided the original author and source are credited. 
and another contraceptive when a couple is not attempting to conceive is the standard recommendation but, it is practiced by a minority of adolescent couples $[4,9,10]$. HIV infected adolescents reported prior use of dual protection during $16 \%$ of their visits [4]. Previous international studies have demonstrated that HIV positive adolescent and adult women are more likely to request injectable contraceptives than their HIV negative peers [4,9,11-13]. Risk of HIV acquisition or progression among hormonal contraception users has been a recent concern based on international trials [14]. The World Health Organization and the Centers for Disease Control consider hormonal contraceptives to be safe and effective options for HIV positive women. This small pilot study is the first to compare contraception practices of perinatally and behaviorally infected adolescent females at one tertiary level public health clinic in the United States.

\section{Materials and Methods}

\section{Study design}

This single-center, cross-sectional survey of HIV positive adolescent females was conducted as a pilot, nested trial. All participants were previously enrolled in a prospective longitudinal cohort study of HIV positive patients at the Maternal Child and Adolescent Center (MCA) for Infectious Disease and Virology, University of Southern California, Keck School of Medicine. All females 15-25 years of age, who had one or more clinic visits from January 1, 2010 to December 31, 2012, were studied. Data was collected by chart review of clinical care that was provided during this time period. Contraceptive practices among perinatally and behaviorally infected adolescents were compared to United States national statistics, (the National Survey of Family Growth and the National Longitudinal Study of Adolescent Health). Secondary outcomes related to previous pregnancies and live births were also analyzed. The University of Southern California Institutional Review Board approved this research.

\section{Study population}

All HIV positive patients, their children, and sexual partners who receive clinical care at MCA are recruited by research staff for participation in a prospective study of HIV transmission, progression and associated health outcomes. Written informed consent is obtained from all patients who agree to participate. Encompassed within this trial are chart review investigations of health indicators, clinical practices and outcomes. This retrospective chart review study was conducted as a nested cohort of adolescent females who were previously enrolled in the MCA longitudinal study.

A consideration of our cohort is that perinatally infected adolescents often receive care at MCA since infancy or childhood. However, the majority of behaviorally acquired adolescents are referred for prenatal care during their pregnancy. The MCA clinic continues to offer all women infectious disease and gynecologic care following their pregnancy. The MCA utilizes an interdisciplinary model to provide a range of services to HIV positive women, men and their families. The patient population is predominately uninsured and or recipients of public assistance. Adolescents at the MCA have the option to continue to receive care from their pediatrician until the age of 25 , but can also receive care with adult and gynecology trained providers. For this reason, females 15 to 25 years of age were included in this study. Females undergoing a work-up for infertility or who were not at risk for pregnancy (mullerian abnormalities, Turners, etc.) were excluded

\section{Study procedure}

All physician, social work and allied provider documents in the medical chart were reviewed for demographic data and information regarding sexual health and contraception utilization.

\section{Statistical analysis}

This was a pilot study to investigate possible differences in contraceptive practices among behaviorally and perinatally infected adolescent females. For comparison the rates of the cohort were listed beside national indices. Data were presented as medians and ranges for continuous data and numbers (n) and percentiles (\%) for categorical data. Differences between the two groups were compared with Wilcoxon Mann-Whitney U test for the continuous data. A chi square contingency table analysis and Fisher's exact probability test, where appropriate, were used to compare the categorical variables between the groups. Due to sparse cell counts with some categorical variables, such as the types of contraceptive, results are listed as a simple descriptive analysis ( $\mathrm{n}$ and \%). All analyses were performed using SAS v9.3. A twosided alternative hypothesis and a 0.05 significance level were assumed in all testing. Subset analysis was performed on adolescents 15 to 19 and 20 to 25 years of age. These age groups are captured in United States National Vital Statistics surveys and reports.

\section{Results}

Of the 33 adolescent females who met the study criteria, 18 (55\%) were perinatally infected and $15(45 \%)$ were behaviorally infected through heterosexual activity. For both groups the mean age at the time of enrollment was 19 years $(\mathrm{p}=0.84)$. Both groups were similar in terms of HIV viral load ( $\mathrm{p}=0.32)$, CD4 count $(\mathrm{p}=0.56)$ and race, with all but one female being Hispanic or Black $(p=0.85)$. Baseline characteristics

\begin{tabular}{|c|c|c|c|}
\hline & Perinatal^ $^{\wedge}(n=18)$ & $\begin{array}{l}\text { Behavioral^^} \\
\quad(n=15)\end{array}$ & P-values \\
\hline Age at enrollment* & $19(15-25)$ & $19(16-22)$ & 0.84 \\
\hline Gravity - pregnancies ${ }^{\star *}$ & & & $<0.0001$ \\
\hline Nulligravid - no pregnancies & $12(67)$ & $0(0)$ & \\
\hline Gravid - previous pregnancy & $6(33)$ & $15(100)$ & \\
\hline Parity - live births ${ }^{* *}$ & & & $<0.0001$ \\
\hline Nulliparous - no live births & $15(83)$ & $1(7)$ & \\
\hline Parous - previous live birth & $3(17)$ & $14(93)$ & \\
\hline \multicolumn{4}{|l|}{ Cumulative lifetime $^{\star \star \star}$} \\
\hline Total pregnancies & 8 & 41 & \\
\hline Total live births & 3 & 28 & \\
\hline Viral load (mean copies $/ \mathrm{mm}^{3}$ ) & 165 (0 to 248154$)$ & 64 (0 to 10676) & 0.32 \\
\hline CD4 (cells/ mm³) & 506 (15 to 1084$)$ & 648 (270 to 867$)$ & 0.56 \\
\hline Race/Ethnicity & & & 0.85 \\
\hline Hispanic white & $11(61)$ & $8(53)$ & \\
\hline Black & $6(33)$ & $7(47)$ & \\
\hline Asian & $1(6)$ & $0(0)$ & \\
\hline Language & & & 1.00 \\
\hline English & $10(56)$ & $8(53)$ & \\
\hline Spanish & $7(39)$ & $7(47)$ & \\
\hline Other & $1(6)$ & $0(0)$ & \\
\hline
\end{tabular}

^Perinatal HIV infection at birth or as a neonate

$\wedge^{\wedge}$ Behavioral HIV infection through heterosexual contact

${ }^{*}$ Mean at enrollment (range), ${ }^{* *}$ Total number of participants (\%)

Wilcoxon rank sum test and Chi square were used with two tailed statistical significance at $5 \%$

${ }^{* * *}$ Cumulative total lifetime pregnancies for all subjects in each group

Table 1: Characteristics of HIV Positive Los Angeles County Adolescent Females Age 15 to 25 from January 1, 2010 to December 31, 2012 


\begin{tabular}{|c|c|c|c|}
\hline & $\begin{array}{l}\text { HIV+ Perinatal^ } \\
\text { N (\%) }\end{array}$ & $\begin{array}{l}\text { HIV+ Behavioral^^ } \\
\text { N (\%) }\end{array}$ & 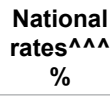 \\
\hline \multicolumn{4}{|c|}{ Progetin only methods } \\
\hline LNG & $1(5.6)$ & $3(20)$ & 2 \\
\hline ENG & $1(5.6)$ & $2(13.3)$ & - \\
\hline DMPA & $7(38.9)$ & $4(26.7)$ & 3.4 \\
\hline \multicolumn{4}{|c|}{ Combined hormonal methods } \\
\hline $\mathrm{COC}$ & $1(5.6)$ & $1(6.7)$ & 45 \\
\hline Patch & $1(5.6)$ & $3(20)$ & 3 \\
\hline Ring & $0(0)$ & $1(6.7)$ & 2 \\
\hline \multicolumn{4}{|c|}{ Non-hormonal methods } \\
\hline Condoms & $4(22.2)$ & $0(0)$ & 29 \\
\hline Abstinence & $3(16.7)$ & $1(6.7)$ & 15 \\
\hline Total & $18(100)$ & $15(100)$ & - \\
\hline
\end{tabular}

${ }^{\wedge}$ Perinatal HIV infection at birth or as a neonate

$\wedge^{\wedge}$ Behavioral HIV infection through heterosexual activity

$\wedge \wedge \wedge$ Percentage of US women age 15-29 based on CDC/NCHS, National Survey of Family Growth, 2006-2010. Other methods such as withdrawal or natural family planning are not listed [15]

LNG: Levonorgestrel-Releasing Intrauterine System (Mirena®), ENG: Etonogestrel Implant (Nexplanon or Implanon $®)$, DMPA: Depot Medroxyprogesterone Acetate COC combined oral contraceptive, Patch (Ortho Evra ${ }^{\circledR}$ ), Ring (Nuva Ring ${ }^{\circledR}$ )

Condoms were the primary contraceptive; persons who used condoms for dual protection are listed according to the hormonal contraceptive utilized.

Table 2: Current Contraceptive Method Used Among HIV Positive Los Angeles County Adolescent Females Age 15 to 25 from January 1, 2010 to December 31, 2012

are listed in Table 1. The majority of participants were English speaking, but $39 \%$ of perinatal and $47 \%$ of behavioral adolescents were Spanishspeaking. The age of sexual debut was not consistently recorded; however, all but two perinatally acquired adolescents reported heterosexual activity by the age of enrollment.

At the end of the study period the perinatal group reported a total of 8 lifetime pregnancies, which occurred among 6 adolescents; the behaviorally infected group reported 41 pregnancies $(\mathrm{p}<0.0001)$. Eighty percent $(n=12)$ of the behaviorally group were referred to MCA with the diagnosis of HIV for the management of their pregnancy. Consequently, the perinatally infected adolescents were more likely to be nulligravid (67\%) than the behaviorally infected adolescents (0\%). Concordantly the majority of behaviorally infected adolescents reported at least one live birth (93\%), as compared to the perinatally infected (17\%).

As per the standard of care all patients were counseled on condom use to prevent transmission and this was documented in all charts. Table 2 lists type of contraception used by behaviorally and perinatally infected groups, as well as U.S. rates for all adolescents in the same age range. Condoms, when used in conjunction with hormonal contraception for dual protection or when used alone as the only means of contraception, were the most commonly utilized method $(n=14$, $42 \%)$. Among the adolescents that reported condom use $(n=14)$, the majority employed a second contraceptive $(n=10)$ and utilized the condom for dual protection (to prevent transmission of HIV and pregnancy). Condom utilization as the primary method and for dual protection was higher among perinatally infected adolescents $(n=9$, $50 \%)$ compared to the behaviorally infected $(\mathrm{n}=5,33 \%)$.

Depo Medroxyprogesterone Acetate (DMPA) was the second most common contraceptive in the cohort, used by 11 adolescents (30\%) of whom 7 (39\%) were perinatally infected and 4 (27\%) were behaviorally infected. Perinatally infected adolescents were more likely to employ abstinence $(n=3,17 \%)$ than the behaviorally infected women $(n=0)$.
Only one perinatally infected adolescent reported utilizing abstinence as her means of contraception after her first coital experience. Long Acting Reversible Contraception (LARC) was utilized by 2 (11\%) of the perinatally infected and $5(33 \%)$ of the behaviorally infected adolescents, both of which are higher than national U.S. rates [5]. Our participants' rate of combined oral contraceptive use (6-7\%) is far less than the national U.S. rate (21.8\%) [15].

Among perinatally infected adolescents, 13 (72\%) changed their contraceptive method during the two year study period. Three $(17 \%)$ reported using contraception at the time of an unintended pregnancy. One woman discontinued contraception to attempt pregnancy, 3 discontinued due to side effects. In the behaviorally infected group, $6(60 \%)$ women changed their contraceptives during the study period. Five discontinued hormonal methods and used condoms due to side effects. One woman utilized 4 different contraceptives during the study period. Three $(30 \%)$ reported using a contraceptive at the time of an unintended pregnancy. Two behaviorally acquired women discontinued contraception because they desired pregnancy.

\section{Discussion}

The unique descriptive results from our cohort may not be generalizable due to the small sample size and potential selection bias. However, there is limited data on contraceptive practices and parity that compare perinatally and behaviorally infected adolescents in the United States. Involvement in this cohort was subject to voluntary enrollment in a prospective longitudinal trial and may reflect adolescents who are more motivated to participate in health care or who have better uptake of medical resources than others who declined enrollment. Strengths of this study include that the same providers, in one clinical setting, cared for all participants with access to similar resources and support services. Due to the retrospective nature of this cross sectional review, our data collection was subject to clinical documentation limitations. Patient reporting was subject to recall bias. Standardized forms were used for clinical encounters, such as gynecology, infectious disease and nutrition, which did stream line data collection and may have mitigated some inter-provider documentation variations.

Another consideration is that adolescents receive sexual health and contraception counseling from various physicians, mid-level providers and other qualified staff at MCA. Nurse practitioners, physician assistants, obstetrician/ gynecologists, pediatricians and internal medicine physicians offered sexual health and contraception services. This approach ensures that adolescents can access a range of providers to address their sexual health issues. However, standardized education can impact a woman's contraceptive choice and practices [16]. As a consequence the contraception utilized by our cohort may reflect the counseling of the individual providers and staff. The demographic of our HIV positive female population at our single United States site may not reflect the same demographics of other regions or countries. Our sample represents adolescents who have access to clinical care, social work support and anti-retroviral therapy. It may be difficult to generalize these results to women who are immuno-compromised, who do not have access to clinicians or who are unable to access contraception or antiretroviral therapy.

Forty four percent of women in the United States age 15-24 are using some method of contraception [15]. Eighty three percent $(n=15)$ of our perinatally infected adolescents and $93 \%(n=14)$ of our behaviorally infected adolescents were using some type of contraception. Fourteen $(42 \%)$ women reported condom usage at some time during the study period, which is far from the recommendation of consistent use with 
every sexual act. This rate is similar to other reports in the literature for HIV positive adolescents and women [4,10,11,17-20]. Tassiopoulos noted that $28 \%$ of perinatally infected adolescents 10 to 18 years of age (males and females) reported sexual intercourse, $62 \%$ had recent intercourse without a condom, and the median age of sexual debut was 14 years of age [20]. He also noted that these rates are similar to the prevalence reported for high school youth with similar demographic backgrounds. Koenig et al surveyed HIV infected boys and girls, $47 \%$ reported a history of unprotected intercourse since their sexual debut (12 perinatal and 37 behavioral), again the average age was 14 [19]. Our findings and the results of previous publications reinforce that adolescents would benefit from further support and sexual health interventions to improve and optimize condom usage so as to reduce the risk of transmission. Sexual debut and intimacy are normal cultural and social developmental milestones that pose a unique challenge to HIV positive adolescents, who must also consider the risks of transmission related to their sexual experinces.

DMPA was the second most commonly used contraceptive, by $39 \%$ $(n=7)$ of our perinatally infected adolescents and $27 \%(n=4)$ of our behaviorally infected adolescents. Globally, DMPA is most commonly used hormonal contraceptive among HIV positive women, and has been show to more effectively prevent unplanned pregnancies among HIV serodiscordant couples than combined oral contraceptive pills [13,21]. Combined Oral Contraceptive pills (COC) are the most commonly used method of contraception among U.S. adolescents, they are used by $22 \%$ of adolescents and $56 \%$ of contraceptors in this age group [15,22]. However, COCs were used infrequently by perinatally infected $(n=1$ or $5.6 \%)$ or behaviorally infected adolescents in our cohort $(\mathrm{n}=1$ or 6.7\%). This may be due to concerns regarding drug interactions, risk for unintended pregnancy, difficulty with adherence, concern about transmission or a high existing pill burden among these young women $[4,5,18]$. Other cohorts of HIV positive women have also demonstrated uptake of hormonal contraceptives that are far less than the national averages [18]. Three adolescents in the perinatal infection group (17\%) and 5 behavioral (33\%) discontinued a hormonal contraceptive due to side effects. Access to contraception choices such as the Implant, Intrauterine Device (IUD), ring, and patch are important among women who cannot comply with daily adherence or have side effects with COCs [9].

Long Acting Reversible Contraception (LARC), such as the implant, hormonal IUD and copper IUD, often offer a better side effect profile, have significantly less risk of unintended pregnancy, and do not require daily adherence. Two perinatally infected adolescents (11\%) and 5 behaviorally infected adolescents (33\%) used the implant or hormonal IUD. LARC is a well-tolerated, cost effective and clinically safe option for adolescents that should be encouraged for HIV positive teens [23]. There were no adverse outcomes, severe reactions or changes in HIV status that were attributed to contraceptives in the clinical records. Other cohorts have noted HIV positive reproductive age women have not mirrored increased LARC uptake among reproductive age women in the US over the last decade; only $2.8 \%$ of HIV + women use a LARC method [18,24]. Further research is needed to assess and remedy barriers to LARC among HIV positive.

The behaviorally infected adolescent group reported significantly higher lifetime live birth and pregnancy histories compared to the perinatally infected adolescents $(\mathrm{p}<0.001)$ and national rates [25]. The behavioral group represents a biased sample that was referred to the MCA for the management of pregnancy. However, the behavioral group also exhibited 41 total lifetime pregnancies among 15 adolescents (range 1-5 pregnancies). By contrast the perinatal group had a total of 8 lifetime pregnancies among 18 adolescents (range 0-2). By age 25 approximately $30 \%$ of women in the U.S. will have had a live birth [25]. Among adolescents age 15-19 in 2006 the national pregnancy rate was 106.7 , the abortion rate was 45.7 per 1,000 women; in the 20-24 age group the rate was 171 and the abortion rate was 39.9 [26]. Due to the limitations of the study investigators were unable to collect detailed pregnancy history information such as: number of pregnancies following HIV diagnosis, pregnancy intervals or outcomes. These pregnancy rates are alarming, previous research has demonstrated the majority of pregnancies reported by HIV infected women are unintended, HIV infected women may have poor access to social resources and may not be in control of sexual negotiations [18].

It is reasonable that adolescents that have contracted HIV through heterosexual intercourse were also vulnerable to unintended pregnancy. Their HIV acquisition risk factors may be the same risk factors that exposed them to adolescent pregnancy such as contraceptive nonadherence, ignorance about pregnancy risk or a lack of reproductive health knowledge. It also may be related to ambivalence about becoming a parent, lack of autonomy in a relationship, peer pressure, poor negotiation skills, or lack of impulse control. By contrast the perinatally infected adolescent group demonstrated mean lifetime live birth and pregnancy rates that were comparable to national averages. Many of them have lost their mother to HIV and have lived their lives with this stigmatizing illness. Other research in this population has demonstrated that pregnancy rates ( 18.8 per 1000 person years or 38 pregnancies among 638 adolescents) were lower among adolescent girls perinatally infected with HIV than among non-HIV infected adolescents age 13-25 [17]. In this same cohort $24 \%$ of girls reported that they were sexually active and none had received antiretroviral therapy prior to or immediately following their birth. Their contraception utilization, and risk reduction behaviors may reflect the education, support, partner disclosure, and contraceptive services they have accessed and their understanding of their diagnosis. Despite the differences in pregnancy rates both groups would benefit from age appropriate behavioral interventions that could optimize their sexual health awareness and behaviors.

The high rates of contractive uptake and LARC utilization among both groups in this cohort probably reflect the gynecologic and sexual health support women are accessing at the MCA. It is imperative that providers emphasize medication adherence and condom use, which are both interventions that will help HIV positive adolescents reduce transmission of the virus. Despite the limitations of this survey it does offer insight into the contraceptive practices, and parity of two very distinct HIV positive adolescent populations. It is imperative that health care providers meet the reproductive health needs of these adolescents because their sexual behaviors will directly impact the risk of disease transmission, progression and prognosis.

\section{Conclusion}

In this small pilot study, perinatally infected adolescents were more likely to use DMPA or condoms as compared to behaviorally infected adolescents, who were more likely to use LARC or DMPA. The reproductive health practices of behaviorally vs. perinatally infected HIV positive adolescent women may reflect different lifestyle and risk taking behaviors. Larger studies should be conducted to explore pregnancy rates, outcomes (abortion, miscarriage, live birth) and contraception utilization among perinatal and behaviorally infected adolescents. These two unique and distinct populations may not mirror our national statistics. 
Citation: Atrio J, Graham K, Vora H, Operskalski E, Kovacs A (2013) Contraceptive use in Adolescents with Perinatally and Behaviorally Acquired Human Immunodeficiency Virus Infection Seen in a Public Los Angeles County Clinic. J AIDS Clin Res 4: 244. doi: 10.4172/2155-6113.1000244

\section{Financial Disclosures and Conflicts of Interest: None to} declare

\section{Acknowledgement}

Staff at Maternal Child Adolescent Center for Infectious Diseases and Virology (MCA) and the patients who have participated in MCA's prospective study.

\section{References}

1. Centers for Disease Control and Prevention (2012) Monitoring selected national HIV prevention and care objectives by using HIV surveillance dataUnited States and 6 US dependent areas-2010. HIV Surveillance Supplemental Report 2012: 17

2. Centers for Disease Control and Prevention (2012) HIV among Pregnan Women, Infants, and Children in the United States.

3. Johnson KB, Akwara P, Rutstein SO, Bernstein S (2009) Fertility preferences and the need for contraception among women living with HIV: the basis for a joint action agenda. AIDS 23 Suppl 1: S7-7S17.

4. Belzer M, Rogers AS, Camarca M, Fuchs D, Peralta L, et al. (2001) Contraceptive choices in HIV infected and HIV at-risk adolescent females. J Adolesc Health 29: 93-100.

5. Kancheva Landolt NT, Lakhonphon S, Ananworanich J (2011) Contraception in HIV-positive female adolescents. AIDS Res Ther 8: 19.

6. Fair C, Wiener L, Zadeh S, Albright J, Mellins CA, et al. (2013) Reproductive health decision-making in perinatally HIV-infected adolescents and young adults. Matern Child Health J 17: 797-808.

7. Obare F, Birungi $H(2010)$ The limited effect of knowing they are HIV-positive on the sexual and reproductive experiences and intentions of infected adolescents in Uganda. Popul Stud (Camb) 64: 97-104.

8. Martinez G, Copen C, Abma J (2011) Teenagers in the United States: Sexual activity, contraceptive use, and childbearing, 2006-2010 National Survery of Family Growth. National Center for Health Statisitcs. Viral Health Stat 23.

9. Say R, Mansour D (2009) Contraceptive choice for young people. J Fam Plann Reprod Health Care 35: 81-85.

10. Birungi H, Mugisha JF, Obare F, Nyombi JK (2009) Sexual behavior and desires among adolescents perinatally infected with human immunodeficiency virus in Uganda: implications for programming. J Adolesc Health 44: 184-187.

11. Heys J, Kipp W, Jhangri GS, Alibhai A, Rubaale T (2009) Fertility desires and infection with the HIV: results from a survey in rural Uganda. AIDS $23 \mathrm{Suppl}$ 1: $\mathbf{S} 37-45$

12. Marlow HM, Maman S, Groves AK, Moodley D (2012) Fertility intent and contraceptive decision-making among HIV positive and negative antenatal clinic attendees in Durban, South Africa. Health Care Women Int 33: 342-358.
13. Whiteman MK, Kissin DM, Samarina A, Curtis KM, Akatova N, et al. (2009) Determinants of contraceptive choice among women with HIV. AIDS 23 Suppl 1: S47-54.

14. Heffron R, Were E, Celum C, Mugo N, Ngure K, et al. (2010) A prospective study of contraceptive use among African women in HIV-1 serodiscordant partnerships. Sex Transm Dis 37: 621-628.

15. Jones J, Mosher W, Daniels K (2012) Current Contraceptive Use in the United States, 2006-2010, and Changes in Patterns of Use Since 1995. National Center for Health Statistics. Vital Health Stat Number.

16. Winner B, Peipert JF, Zhao Q, Buckel C, Madden T, et al. (2012) Effectiveness of long-acting reversible contraception. N Engl J Med 366: 1998-2007.

17. Brogly SB, Watts DH, Ylitalo N, Franco EL, Seage GR 3rd, et al. (2007) Reproductive health of adolescent girls perinatally infected with HIV. Am J Public Health 97: 1047-1052.

18. Massad LS, Evans CT, Wilson TE, Golub ET, Sanchez-Keeland L, et al. (2007) Contraceptive use among U.S. women with HIV. J Womens Health (Larchmt) 16: $657-666$

19. Koenig LJ, Pals SL, Chandwani S, Hodge K, Abramowitz S, et al. (2010) Sexual transmission risk behavior of adolescents With HIV acquired perinatally or through risky behaviors. J Acquir Immune Defic Syndr 55: 380-390.

20. Tassiopoulos K, Moscicki A, Mellins C, Kacnnek D, Malee K, et al. (2013) Sexual risk behavior among youth with perinatal HIV infection in the United States: predictors, implications for intervention development. Clinical Infectious Diseases 56: 283-290.

21. Ngure K, Heffron R, Mugo N, Celum C, Cohen C, et al. (2012) Contraceptive method and pregnancy incidence among women in HIV-1 serodicordant partnerships. AIDS 26: 513-518.

22. Abma J, Martinez G, Copen C. (2011) Teenagers in the United States: Sexual activity, contraceptive use, and childbearing, 2006-2010 National Survery of Family Growth. National Center for Health Statisitcs. Viral Health Stat 23.

23. Verhaeghe $\mathrm{J}$ (2012) Clinical practice: Contraception in adolescents. Eur J Pediatr 171: 895-899.

24. Sun M, Peipert JF, Zhao Q, Wilson TE, Weber KM, et al. (2012) Trends in contraceptive use among women with human immunodeficiency virus. Obste Gynecol 120: 783-790.

25. Martinez G, Daniels K, Chandra A (2012) Fertility of men and women aged 15 44 years in the United States: National Survey of Family Growth, 2006-2010. Natl Health Stat Report : 1-28.

26. Kost K, Henshaw S, Carlin L (2013) U.S. teenage pregnancies, births and abortions; National and state trends and trends by race and ethnicity. Guttmacher Institute. 